\title{
Diagnostica dell'insufficienza renale acuta e biomarkers di danno renale
}

\author{
Paolo Lentini ${ }^{1}$, Massimo de Cal ${ }^{1}$, Luca Zanoli ${ }^{2}$, Antonio Granata ${ }^{3}$, Roberto Dell'Aquila ${ }^{1}$ \\ ${ }^{1}$ UOC Nefrologia e Dialisi, Ospedale San Bassiano, Bassano del Grappa (VI) \\ ${ }^{2}$ Dipartimento di Medicina Interna, Università degli Studi di Catania, Catania \\ ${ }^{3}$ UOC Nefrologia e Dialisi, Ospedale San Giovanni di Dio, Agrigento
}

\begin{abstract}
Acute Kidney Injury: the role of biomarkers for early diagnosis
Acute kidney injury is an important health problem and its incidence is increasing. Acute kidney injury has traditionally been measured and defined using surrogate measurements of the function of renal filtration, as plasma creatinine and urea. Despite the serious delay in diagnosing acute kidney injury, creatinine remains the most used biomarker. In recent years, clinicians highlighted the need to find new biomarkers for acute kidney injury that can replace or implement the data found using creatinine and that can allow an early measurement of the damage. Despite the multiple biomarkers analyzed and tested in recent years, creatinine is the reference marker because it is easy to measure and for its lower costs.

Biomarker panels, employed in various frames help to determine the beginning and the duration of kidney damage or the severity of acute kidney injury, may assist in the diagnosis of acute kidney injury.
\end{abstract}

Keywords: Acute kidney injury, Biomarkers, Creatinine

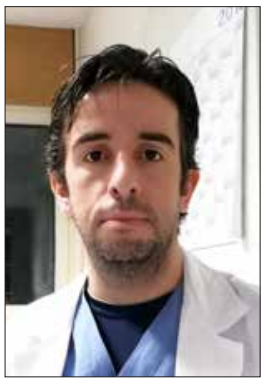

L'Insufficienza Renale Acuta (IRA) rappresenta un problema che ha riscosso una crescente attenzione nel corso degli ultimi anni nella pratica clinica. Nonostante i progressi in campo terapeutico la mortalità, la morbilità e i costi ospedalieri associati all'IRA rimangono assai elevati.

L'IRA rappresenta, quindi, un importante problema sanitario e la sua incidenza è in aumento soprattutto nei pazienti con problemi cardiovascolari. Le percentuali di IRA variano dal $5 \%$ dei pazienti ospedalizzati fino al $30-50 \%$ dei pazienti di cardiochirurgia o di terapia intensiva (1-4).

L'IRA è stata tradizionalmente rilevata e definita mediante

Accepted: February 27, 2016

Published online: April 18, 2016

Indirizzo per la corrispondenza:

Dr. Paolo Lentini

UOC di Nefrologia e Dialisi

Ospedale San Bassiano

Via Dei Lotti 40

36061 Bassano del Grappa (VI)

paolo.lentini@yahoo.it la misurazione di surrogati della funzione di filtrazione renale, come creatinina plasmatica e urea.

Per soddisfare la necessità di una definizione uniforme per identificare e classificare la gravità dell'IRA viene ideata la classificazione RIFLE. Tale classificazione si basa su 5 livelli:

Risk: aumento della creatinina di 1.5 volte, riduzione della velocità di filtrazione glomerulare del $25 \%$ o riduzione della diuresi a meno di $0.5 \mathrm{~mL} / \mathrm{kg}$ per $6 \mathrm{~h}$.

Injury: aumento della creatinina di due volte, riduzione della velocità di filtrazione glomerulare del $50 \%$ o riduzione della diuresi a meno di $0.5 \mathrm{~mL} / \mathrm{kg}$ per $12 \mathrm{~h}$.

Failure: aumento della creatinina di tre volte, riduzione della velocità di filtrazione glomerulare del $75 \%$ o riduzione della diuresi a meno di $0.5 \mathrm{~mL} / \mathrm{kg}$ per $24 \mathrm{~h}$ o anuria per $12 \mathrm{~h}$.

Loss: perdita completa della funzione renale che richiede la dialisi per più di 4 settimane.

End-Stage Kidney Disease: uremia che richiede la dialisi per più di 3 mesi.

L'aumento della creatinina, secondo la classificazione RIFLE, andrebbe monitorato per un periodo di 7 giorni (2).

$\mathrm{Nel}$ 2007, viene creata una versione modificata della RIFLE, denominata AKIN (Acute Kidney Injury Network), nella quale si definiscono i criteri dell'IRA in base a:

STADIO 1: i livelli di creatinina aumentano di $0.3 \mathrm{mg} / \mathrm{dL}$ in $48 \mathrm{~h}$ o aumentano dal 150 al $200 \%$ o riduzione della diuresi a meno di $0.5 \mathrm{~mL} / \mathrm{kg} / \mathrm{h}$ per $6 \mathrm{~h}$.

STADIO 2: i livelli di creatinina aumentano dal 200 al 300\% 
o riduzione della diuresi a meno di $0.5 \mathrm{~mL} / \mathrm{kg} / \mathrm{h}$ per $12 \mathrm{~h}$.

STADIO 3: i livelli di creatinina aumentano oltre il $300 \%$ o riduzione della diuresi a meno di $0.3 \mathrm{~mL} / \mathrm{kg} / \mathrm{h}$ per $24 \mathrm{~h} \mathrm{o}$ anuria per $12 \mathrm{~h}$. Tutti i pazienti in terapia sostitutiva rientrano nello STADIO 3.

Si passa, quindi, dai 5 livelli della classificazione RIFLE ai 3 STADI della classificazione $\operatorname{AKIN}(5,6)$; entrambe le classificazioni si basano sui livelli sierici della creatinina e sull'output urinario, che definiscono gli stadi di disfunzione renale (7) e, certamente, la creatinina è il marker più diffuso per la funzione renale, in quanto presenta bassi costi e viene analizzata facilmente.

La misurazione della creatinina sierica come unico parametro di funzionalità renale ha spesso responsabilità oggettive nella progressione del danno renale in quanto è un indicatore tardivo e non specifico essendo influenzato da molteplici fattori come età, sesso, razza e massa corporea (8); inoltre, i livelli di creatinina aumentano in condizioni di febbre, trauma e ipertiroidismo e diminuiscono in condizioni di età avanzata e diminuzione della massa muscolare e in presenza di malattie epatiche.

Se si comparano i marker cardiaci con quelli renali, si può constatare come, negli ultimi 50 anni, vi sia stata un'evoluzione con l'utilizzo di marker cardiaci diversi che ha portato a una diminuzione significativa della mortalità, pari a circa il 50\%, mentre, per quanto riguarda il danno renale, si è fatto sempre e solo affidamento sulle misure condotte sulla creatinina.

Vi è, quindi, la necessità di nuovi marker per l'IRA che permettano di sostituire o implementare il dato rilevato mediante la creatinina; non solo, ma che permettano anche una misurazione precoce del danno.

Una diagnosi precoce di IRA permette, infatti, un intervento terapeutico immediato e può ridurre la progressione della malattia.

Per questo, alcuni nuovi biomarker di danno precoce stanno entrando nello screening diagnostico. In generale, un marker ideale deve essere generato dalle cellule danneggiate, deve essere sensibile e specifico per il danno d'organo, proporzionale all'entità del danno ed espresso precocemente, deve diminuire al termine dell'acuzie ed essere facile e rapido nella misurazione e, per quanto possibile, deve essere non invasivo per il paziente. Tra i molti biomarker in studio, solo alcuni sono stati validati e possono già rivestire un ruolo di sicura utilità nella pratica clinica (9).

Già nel 2007, Honore PM et al. concludevano che era giunto il momento di escludere la creatinina come marker per I'IRA e che risultava essenziale sviluppare dei kit di biomarker in cui combinare diversi marker precoci con caratteristiche differenti $(10,11)$.

Negli ultimi anni, vi è stata la ricerca di nuovi marker per I'IRA prodotti da una serie di geni la cui espressione è attivata entro poche ore dall'insulto renale.

Nel 2008, Vaidya et al. (12) proposero una serie di poten- ziali biomarker urinari che segnalavano l'IRA e la diminuzione del GFR molto prima della creatinina, in quanto erano in grado di segnalare direttamente il danno renale.

Molti di questi biomarker sono stati poco considerati o, addirittura, abbandonati durante gli ultimi anni, ma vi è stata certamente una continua ricerca di nuovi biomarker in grado di soppiantare l'utilizzo della creatinina nella determinazione dell'IRA.

Tra i biomarker, quello, al momento, di maggiore interesse e con più promettenti caratteristiche di precocità e sensibilità è, senza dubbio, il NGAL.

NGAL: è un polipeptide con ponte disolfuro di 178 aa (22 kDa), che, nella forma glicosilata, aumenta fino a $25 \mathrm{kDa}$ (13-15).

Questo polipeptide può essere misurato sia su sangue che su urine ed è in grado, secondo diversi studi recenti, di segnalare il danno renale acuto già poche ore dopo l'evento.

Diversi studi hanno valutato e verificato l'accuratezza diagnostica, mediante l'analisi ROC, della misurazione di NGAL sia nelle urine che nel plasma come marker IRA.

In uno studio del 2008 di Bennett $\mathrm{M}$ et al., condotto su bambini che avevano subito un cardio-pulmonary bypass, il NGAL urinario aumentava di 15 volte dopo 2 ore e di 25 volte dopo 4 ore nei pazienti con IRA, mentre la creatinina sierica aumentava solo dopo 48 ore.

Questo dato è stato confermato anche in ulteriori studi nei quali è stato valutata l'efficacia del NGAL urinario e plasmatico nel predire I'IRA sia in pazienti adulti che in bambini dopo chirurgia cardiaca.

II NGAL plasmatico si è, inoltre, dimostrato un marker precoce non solo nel diagnosticare I'IRA, ma anche nel predire la necessità di Renal Replacement Therapy (RRT). Inoltre, in pazienti con insufficienza renale cronica, NGAL caratterizza molto bene l'entità del danno renale e rappresenta un indicatore indipendente di rischio per la progressione dell'insufficienza renale cronica (16).

NGAL può essere determinato mediante diverse metodiche, sia su plasma che su urine.

Come si evince dalla Tabella proposta da Clerico A et al. (17), i tempi per l'ottenimento dei risultati variano da pochi minuti fino a 24 ore in base alla tecnica utilizzata.

Non vi è un consenso generale sul tipo di campione su cui effettuare il dosaggio, ma, essendo influenzata anche da fattori extrarenali, la misurazione effettuata sulle urine sembra fornire una stima più specifica del danno tubulare renale (18).

I risultati di una recente meta-analisi indicano, comunque, che i campioni siero-plasma e quelli urinari hanno un'accuratezza diagnostica simile per l'IRA (19).

Tra i nuovi marker per I'IRA, che hanno riscosso interesse negli ultimi anni, anche la cistatina $C$ può essere utilizzata per determinare il danno precoce renale.

Cistatina C: è una proteina di $13 \mathrm{kDa}$ non glicosilata, 
inibitrice delle proteasi cisteiniche, che viene prodotta e rilasciata nel sangue con una velocità costante da tutte le cellule nucleate.

Viene filtrata a livello glomerulare, riassorbita e completamente catabolizzata dai tubuli renali.

A differenza della creatinina, non viene influenzata da età, sesso e razza e dalla massa muscolare (20).

La cistatina $C$ può essere impiegata come marker del danno renale precoce; inoltre, risulta utile nel calcolo della funzionalità renale (GFR), in quanto i livelli sierici sono un indice sensibile della funzione renale. All'aumentare dei valori di cistatina C, diminuisce il GFR e viceversa. In uno studio del 2005 veniva dimostrata l'affidabilità della cistatina $\mathrm{C}$ come marker in tempo reale del GFR (21).

La cistatina $C$ si è dimostrata, in diversi studi che coinvolgevano pazienti della terapia intensiva (cirrosi epatica, insufficienza cardiaca cronica, insufficienza respiratoria, chirurgia valvolare, sepsi), un marker dell'IRA superiore alla creatinina.

La cistatina C sembra rilevare I'IRA 1-2 giorni prima della creatinina e aumenta all'approssimarsi del danno renale e in concomitanza con la gravità dell'IRA, secondo la classificazione RIFLE (22).

Shlipak MG et al. in uno studio del 2005 (23) sul rischio di morte e di eventi cardiovascolari in pazienti anziani affermavano la capacità della cistatina $\mathrm{C}$ di predire tali eventi nei pazienti della terapia intensiva (24).

La cistatina C urinaria non solo sembra prevedere precocemente I'IRA ma risulterebbe anche un marker per la mortalità nei pazienti in terapia intensiva. Risulterebbe essere, inoltre, un marker ideale da utilizzare nei pannelli di biomarker per diagnosticare I'IRA (25).

In uno studio prospettico di biomarker sierici per predire I'IRA in pazienti cardiochirurgici, i valori rilevati per NGAL e cistatina $\mathrm{C}$ risultano migliori rispetto ai marker convenzionali nell'identificare I'IRA (26).

La cistatina $C$ può essere misurata sia su sangue che su urine, come NGAL, mediante metodiche diverse; in generale, il metodo nefelometrico è quello più impiegato per determinare la cistatina $\mathrm{C}$.

KIM-1: è una glicoproteina transmembrana con un piccolo dominio intracellulare e un grande dominio extracellulare.

In condizioni normali, non viene espressa dai reni; viene, invece, sovraespressa in risposta a un danno cellulare dai tubuli renali.

A differenza dei marker succitati, la KIM-1 è misurabile solo sulle urine.

Sembra più specifica di NGAL per danni renali nefrotossici o ischemici e non viene influenzata da infezioni urinarie e Chronic Kidney Disease.

La KIM-1 può essere misurata mediante ELISA e sembra essere correlata con la gravità del danno renale.

Nel 2009, in un'analisi comparativa con 6 diversi biomar- ker urinari per effettuare una diagnosi precoce di IRA, KIM-1 è risultata essere il marker migliore dopo 2 ore dal cardiopulmonary bypass (27).

In uno studio del 2010 di Liang XL et al. (28), è stata evidenziata l'importanza di utilizzare in modo combinato i marker urinari KIM-1 e IL-18 per diagnosticare precocemente I'IRA nei pazienti che hanno subito un cardio-pulmonary bypass, in quanto KIM-1 rileverebbe la presenza dell'IRA, mentre IL-18 segnalerebbe lo sviluppo dell'IRA stessa nel paziente.

IL-18: è una citochina pro-infiammatoria con un peso di $18 \mathrm{kDa}$, che viene prodotta dalle cellule tubulari renali e dai macrofagi.

Questa citochina viene coinvolta in vari processi tra i quali l'apoptosi, l'ischemia, il rigetto d'organo, le infezioni e i tumori.

IL-18 può essere misurata sia nel plasma/siero sia nelle urine, ma, misurata nella matrice urinaria, è risultata essere un test affidabile per effettuare una diagnosi precoce di IRA nei pazienti critici sia adulti che bambini e sembrerebbe predire, inoltre, la mortalità (29). Inoltre risulterebbe associata al peggioramento dell'IRA severa (30).

La combinazione di NGAL e di IL-18 analizzati nelle urine consentirebbe la previsione di IRA molto prima della creatinina e, inoltre, essi sembrerebbero aumentare in tandem (31).

Livelli di IL-18 e NGAL entrambi urinari sono associati allo sviluppo di IRA severa e prevedono la necessità di una RRT. Nello studio svolto da Parikh CR et al. (32), risulterebbe marginale l'abilità nel classificare I'IRA mediante questi due marker.

IL-18 urinaria, secondo uno studio di Haase $M$ et al. del 2008, non sarebbe in grado di predire I'IRA nei pazienti che hanno subito un intervento di cardiochirurgia, ma risulterebbe un marker di infiammazione associata a un cardio-pulmonary bypass (33).

Purtroppo, tra i vari nuovi biomarker, non è stato possibile identificarne alcuno che sia potenzialmente in grado di identificare I'IRA in modo specifico.

Dal 2007, si è ipotizzata la creazione di pannelli diversi da impiegare per determinare l'inizio e la durata del danno renale o i livelli di gravità dell'IRA.

Ovviamente, tali pannelli sono stati pensati per identificare I'IRA nelle diverse matrici: nel plasma/siero mediante l'utilizzo dei marker NGAL e cistatina C e nelle urine, utilizzando NGAL, IL-18 e KIM-1.

I pannelli possono essere diversificati anche in base alla funzione che potrebbero svolgere: si possono, infatti, ottenere pannelli per l'identificazione dell'IRA mediante cistatina C, IL-18 e KIM-1 o per diagnosticare precocemente l'IRA utilizzando NGAL e cistatina C o per determinare il rischio di morte o di necessità di RRT mediante NGAL, KIM-1 e IL-18.

Inoltre, utilizzando i valori di riferimento dei vari biomarker nelle varie matrici, è possibile diagnostica I'IRA anche precocemente (Tab. I). 
TABELLA I - Matrice e valori di riferimento dei principali Biomarker per l'insufficienza renale acuta

\begin{tabular}{llc}
\hline Biomarker & Matrice & Valori di riferimento \\
\hline NGAL & Plasma & $>150 \mathrm{ng} / \mathrm{mL}$ \\
NGAL & Urine & $>150 \mathrm{ng} / \mathrm{mL}$ \\
Cistatina C & Plasma & $>1.0 \mathrm{mg} / \mathrm{L}$ \\
Cistatina C & Urine & $>0.5 \mathrm{mg} / \mathrm{L}$ \\
IL-18 & Urine & $>20 \mathrm{pg} / \mathrm{mL}$ \\
KIM-1 & Urine & $>500 \mathrm{pg} / \mathrm{mL}$ \\
\hline
\end{tabular}

\section{Conclusioni}

In conclusione, possiamo affermare che, ancora oggi, la creatinina rimane il marker più utilizzato, nonostante il grave ritardo nel diagnosticare I'IRA.

Certamente, per la facilità di analisi e i bassi costi, rimane il marker di riferimento pur manifestando gravi limitazioni legate anche al sesso del paziente, all'età e alla massa muscolare.

La ricerca e la necessità di identificare nuovi marker per I'IRA sono ancora, però, legate al costo troppo elevato, soprattutto se paragonato alla creatinina.

NGAL, certamente, è stato e rimane il biomarker più studiato negli ultimi anni e sembra poter essere impiegato non solo nell'identificazione dell'IRA ma anche per rilevare i livelli di gravità dell'IRA stessa.

Certamente, i dati forniti da NGAL migliorano e avvalorano i risultati della sola creatinina.

La cistatina $C$ potrebbe essere utilizzata per la determinazione del GFR e per monitorare la gravità dell'IRA.

KIM-1 e IL-18, se utilizzate insieme al NGAL urinario, possono fornire un dato più certo per identificare I'IRA rispetto al risultato che si otterrebbe se prese singolarmente.

La creazione di pannelli di biomarker, impiegati nelle diverse matrici per determinare l'inizio e la durata del danno renale o i livelli di gravità dell'insufficienza renale acuta, potrebbe essere di ausilio nelle diagnostica dell'insufficienza renale acuta, anche precocemente.

\section{Disclosures}

Financial support: No financial support was received for this submission.

Conflict of interest: The authors have no conflict of interest.

\section{Bibliografia}

1. McCullough PA, Shaw AD, Haase M, et al. Diagnosis of acute kidney injury using functional and injury biomarkers: workgroup statements from the tenth Acute Dialysis Quality Initiative Consensus Conference. Contrib Nephrol 2013;182:13-29.

2. Bellomo R, Ronco C, Kellum JA, Mehta RL, Palevsky P. Acute Dialysis Quality Initiative workgroup. Acute renal failure definition, outcome measures, animal models, fluid therapy and information technology needs: the Second International Consensus Conference of the Acute Dialysis Quality Initiative (ADQI) Group. Crit Care 2004;8:R204-12.

3. Walther CP, Podoll AS, Finkel KW. Summary of clinical practice guidelines for acute kidney injury. Hosp Pract 2014;42:7-14.

4. Pickering JW, Endre ZH. The definition and detection of acute kidney injury. J Renal Inj Prev 2013;30:21-5.

5. Mehta RL, Kellum JA, Shah SV, et al. Acute Kidney Injury Network: report of an initiative to improve outcomes in acute kidney injury. Crit Care 2007;11:R31.

6. Levin A, Warnock DG, Mehta RL, et al. Improving outcomes from acute kidney injury: report of an initiative. Am J Kidney Dis 2007;50:1-4.

7. Bagshaw SM, George C, Bellomo R. A comparison of the RIFLE and AKIN criteria for acute kidney injury in critically ill patients. Nephrol Dial Transplant 2008;23:1569-74.

8. Star RA. Treatment of acute renal failure. Kidney Int 1998;54: 1817-31.

9. Emdin $M$, Vittorini $S$, Passino $C$, Clerico $A$. Old and new biomarkers of heart failure. Eur J Heart Fail 2009;11:331-5.

10. Honore PM, Joannes-Boyau O, Boer W, Janvier G, Gressens B. Acute kidney injury in the ICU: time has come for an early biomarker kit! Acta Clin Belg 2007;62(Suppl. 2):318-21.

11. Honore PM, Joannes-Boyau O, Boer W. The early biomarker of acute kidney injury: in search of the Holy Grail. Intensive Care Med 2007;33:1866-8.

12. Vaidya VS, Ferguson MA, Bonventre JV. Biomarkers of acute kidney injury. Annu Rev Pharmacol Toxicol 2008;48:463-93.

13. Paragas N, Qiu A, Hollmen M, Nickolas TL, Devarajan P, Barasch J. NGAL-Siderocalin in kidney disease. Biochim Biophys Acta 2012;1823:1451-8.

14. Flower DR, North AC, Sansom CE. The lipocalin protein family: structural and sequence overview. Biochim Biophys Acta 2000;1482:9-24.

15. Virzì GM, Clementi A, de Cal M, Cruz DN, Ronco C. Genomics and biological activity of neutrophil gelatinase-associated lipocalin in several clinical settings. Blood Purif 2013;35:139-43.

16. Bolignano D, Lacquaniti A, Coppolino G, et al. Neutrophil Gelatinase-Associated Lipocalin (NGAL) and Progression of Chronic Kidney Disease. Clin J Am Soc Nephrol 2009;4:337-44.

17. Clerico A, Galli C, Giovannini S, Cantinotti M, Storti S. NGAL come Biomarcatore di danno renale acuto: il punto di vista del Laboratorio. LigandAssay 2011;16.

18. Mishra J, Dent C, Tarabishi R, et al. Neutrophil gelatinaseassociated lipocalin (NGAL) as a biomarker for acute renal injury after cardiac surgery. Lancet 2005;365:1231-8.

19. Haase M, Bellomo R, Devarajan P, Schlattmann P, Haase-Fielitz A. Accuracy of neutrophil gelatinase-associated lipocalin (NGAL) in diagnosis and prognosis in acute kidney injury: a systematic review and meta-analysis. Am J Kidney Dis 2009;54:1012-24.

20. Zhu J, Yin $\mathrm{R}, \mathrm{Wu} \mathrm{H}$, et al. Cystatin $\mathrm{C}$ as a reliable marker of renal function following heart valve replacement surgery with cardiopulmonary bypass. Clin Chim Acta 2006;374:116-21.

21. Villa $P$, Jiménez $M$, Soriano $M C$, Manzanares J, Casasnovas P. Serum cystatin $C$ concentration as a marker of acute renal dysfunction in critically ill patients. Crit Care 2005;9:R139-43.

22. Herget-Rosenthal S, Marggraf G, Hüsing J, et al. Early detection of acute renal failure by serum cystatin C. Kidney Int 2004;66:1115-22.

23. Shlipak MG, Sarnak MJ, Katz R, et al. Cystatin C and the risk of death and cardiovascular events among elderly persons. $\mathrm{N}$ Engl J Med 2005;352:2049-60.

24. Nejat M, Pickering JW, Walker RJ, et al. Urinary cystatin C is diagnostic of acute kidney injury and sepsis, and predicts mortality in the intensive care unit. Crit Care 2010;14:R85. 
25. Koyner JL, Bennett MR, Worcester EM, et al. Urinary cystatin $\mathrm{C}$ as an early biomarker of acute kidney injury following adult cardiothoracic surgery. Kidney Int 2008;74:1059-69.

26. Haase-Fielitz A, Bellomo R, Devarajan P, et al. Novel and conventional serum biomarkers predicting acute kidney injury in adult cardiac surgery--a prospective cohort study. Crit Care Med 2009;37:553-60.

27. Liangos $\mathrm{O}$, Tighiouart $\mathrm{H}$, Perianayagam MC, et al. Comparative analysis of urinary biomarkers for early detection of acute kidney injury following cardiopulmonary bypass. Biomarkers 2009;14:423-31.

28. Liang $\mathrm{XL}$, Liu SX, Chen $\mathrm{YH}$, et al. Combination of urinary kidney injury molecule-1 and interleukin-18 as early biomarker for the diagnosis and progressive assessment of acute kidney injury following cardiopulmonary bypass surgery: a prospective nested case-control study. Biomarkers 2010;15: 332-9.
29. Parikh CR, Abraham E, Ancukiewicz M, Edelstein CL. Urine IL18 is an early diagnostic marker for acute kidney injury and predicts mortality in the intensive care unit. J Am Soc Nephrol 2005;16:3046-52.

30. Washburn KK, Zappitelli M, Arikan AA, et al. Urinary interleukin-18 is an acute kidney injury biomarker in critically ill children. Nephrol Dial Transplant 2008;23:566-72.

31. Parikh CR, Mishra J, Thiessen-Philbrook H, et al. Urinary IL-18 is an early predictive biomarker of acute kidney injury after cardiac surgery. Kidney Int 2006;70:199-203.

32. Parikh CR, Coca SG, Thiessen-Philbrook H, et al. Postoperative biomarkers predict acute kidney injury and poor outcomes after adult cardiac surgery. J Am Soc Nephrol 2011;22:1748-57.

33. Haase $M$, Bellomo $R$, Story $D$, Davenport $P$, Haase-Fielitz A. Urinary interleukin-18 does not predict acute kidney injury after adult cardiac surgery: a prospective observational cohort study. Crit Care 2008;12:R96. 